\title{
Insights into complication rates, reoperation rates, and healthcare utilization associated with use of recombinant human bone morphogenetic protein-2 in patients with spine infections
}

\author{
Mayur Sharma, MD, MCh, ${ }^{1}$ Nicholas Dietz, MD, ${ }^{1}$ Ahmad Alhourani, MD, ${ }^{1}$ \\ Beatrice Ugiliweneza, PhD, MSPH, ${ }^{1}$ Dengzhi Wang, MS, ${ }^{1}$ Doniel Drazin, MD, ${ }^{2}$ and \\ Maxwell Boakye, MD, MPH, MBA ${ }^{1}$ \\ 'Department of Neurosurgery, University of Louisville, Louisville, Kentucky; and ²Evergreen Hospital Neuroscience Institute, \\ Kirkland, Washington
}

\begin{abstract}
OBJECTIVE Use of recombinant human bone morphogenetic protein-2 (rhBMP-2) in patients with spine infections is controversial. The purpose of this study was to identify long-term complications, reoperations, and healthcare utilization associated with rhBMP-2 use in patients with spine infections.

METHODS This retrospective study extracted data using ICD-9/10 and CPT codes from MarketScan (2000-2016). Patients were dichotomized into 2 groups (rhBMP-2, no rhBMP-2) based on whether rhBMP-2 was used during fusion surgery for spinal infections. Outcomes of interest were reoperation rates (index level, other levels), readmission rates, discharge disposition, length of stay, complications, and healthcare resource utilization at the index hospitalization and 1 , $3,6,12$, and 24 months following discharge. Outcomes were compared using nonparametric 2-group tests and generalized linear regression models.
\end{abstract}

RESULTS The database search identified 2762 patients with $>24$ months' follow-up; rhBMP-2 was used in $8.4 \%$ of their cases. The patients' median age was 53 years, $52.43 \%$ were female, and $15.11 \%$ had an Elixhauser Comorbidity Index $\geq 3$. Patients in the rhBMP-2 group had higher comorbidity indices, incurred higher costs at index hospitalization, were discharged home in most cases, and had lower complication rates than those in the no-rhBMP-2 group. There was no statistically significant between-groups difference in complication rates 1 month following discharge or in reoperation rates at 3,6, 12, and 24 months following the procedure. Patients in the no-rhBMP-2 group incurred higher utilization of outpatient services and medication refill costs at 1, 3, 6, 12, and 24 months following surgery.

CONCLUSIONS In patients undergoing surgery for spine infection, rhBMP-2 use was associated with lower complication rates and higher median payments during index hospitalization compared to cases in which rhBMP-2 was not used. There was no significant between-groups difference in reoperation rates (index and other levels) at 3, 6, 12, and 24 months after the index operation. Patients treated with rhBMP-2 incurred lower utilization of outpatient services and overall payments. These results indicate that rhBMP-2 can be used safely in patients with spine infections with costeffective utilization of healthcare resources and without an increase in complications or reoperation rates.

https://thejns.org/doi/abs/10.3171/2018.10.FOCUS18448

KEYWORDS long-term; outcomes; healthcare utilization; BMP-2; spine infections

$\mathrm{V}$ ERTEBRAL osteomyelitis (VO) is commonly seen in patients with underlying medical disorders such as diabetes mellitus and those who use immunosuppressive agents, intravenous drugs, and opioids. ${ }^{7}$ Urinary tract infection is also a common source of spine infec- tion..$^{14}$ Neurological deficits can be seen in up to $32 \%$ of patients with VO. ${ }^{14}$ Diagnosis is often possible following CT-guided or open biopsy of the lesion (77\%) or blood cultures (58\%). ${ }^{14}$ Staphylococcus aureus is the most common organism identified in pyogenic $\mathrm{VO} .{ }^{3,14}$ However, various

ABBREVIATIONS ECI = Elixhauser Comorbidity Index; ED = emergency department; IQR = interquartile range; $\mathrm{OR}$ = odds ratio; rhBMP-2 = recombinant human bone morphogenetic protein-2; RR = relative risk; $\mathrm{VO}=$ vertebral osteomyelitis.

SUBMITTED August 27, 2018. ACCEPTED October 30, 2018.

INCLUDE WHEN CITING DOI: 10.3171/2018.10.FOCUS18448. 
fungal, mycobacterial, nocardial, and Brucella species are identified in rare cases involving patients with intravenous drug abuse. ${ }^{1,3,7,11,15}$ Prolonged antibiotic therapy, for at least 6-8 weeks if the infection is bacterial or lifelong if it is fungal or mycobacterial, is indicated in these patients. However, despite aggressive treatment, relapse of infection and patient death have been reported in up to $32 \%$ and $6 \%-15 \%$ of cases, respectively. ${ }^{4,14}$ Patients with microbiologically negative VO had less systemic inflammatory response and better clinical outcomes than those with microbiologically positive $\mathrm{VO}$ with at least 8 weeks of antibiotics. ${ }^{9,12}$ Surgical decompression and fusion is necessary in a subset of patients with VO and epidural abscess, with bony destruction necessitating spinal fusion for instability. ${ }^{2,3}$ Use of recombinant human bone morphogenetic protein-2 (rhBMP-2) in these procedures has been motivated by the need to have improved fusion rates after extensive spinal destruction by the infection and surgical decompression.

The rhBMP-2 allograft in spinal fusion has been widely used since its clinical inception in 2002 and has demonstrated improved fusion rates (posterolateral fusion rate of $88 \%$ ) in comparison to traditional iliac crest bone graft $(73 \%) .{ }^{13}$ In 2006, it was estimated that BMP was used in $25 \%$ of all spinal fusion procedures in the United States. ${ }^{5}$ Its use in the setting of spine infection, however, remains controversial. A retrospective study showed that rhBMP-2 had been successfully used in spinal fusion during active infection without complication for 20 months postoperatively. ${ }^{3}$ In the setting of pyogenic VO, rhBMP-2 has been shown to be an effective treatment, with solid bone fusion, clinical improvement, and no recurrence of infection or instrumental failure before 2 years. ${ }^{2,3}$ Additionally, evidence of rhBMP-2 in other osseous infections shows promise for spine surgery, as the reduction rate of infection of $48 \%$ has been observed in type III tibia fractures with use of rhBMP-2. ${ }^{20}$ However, the evidence in favor of rhBMP-2 use in patients with spine infections is from small retrospective studies and there is no study investigating this question using a large database.

In the present study, we examine the use of rhBMP-2 in spine infection in patients extracted from the national MarketScan database (2000-2016) to determine rates of reoperation, readmission, discharge, length of stay, complications, and healthcare resource utilization. We hypothesize that rhBMP-2 will reduce complications that necessitate reoperation and readmission, and will decrease healthcare resource utilization. Safe and effective use of rhBMP-2 in patients with VO may enhance health outcomes during spine infection while also limiting healthcare utilization postoperatively.

\section{Methods}

\section{Patient Selection}

We used the inpatient admission tables from the national MarketScan database (2000-2016) to extract data on patients with a primary diagnosis of spinal infection with a concurrent procedure of fusion and $>12$ months of follow-up. These patients were stratified into 2 groups based on whether or not rhBMP-2 was used during fu- sion for spine infections. These 2 groups are termed the 1) rhBMP-2 group and 2) no-rhBMP-2 group. Exclusion criteria included concurrent re-fusion (at additional levels other than the index level of interest) or patient age less than 18 years. To screen for diagnosis, procedure, and BMP use, we used ICD-9, ICD-10, and CPT-4 codes. For each patient, the first occurring case satisfying the above conditions was flagged as the index hospitalization.

Patients with $>24$ months of follow-up were identified and data were analyzed for both that group and the overall group of patients with $>12$ months of follow-up.

\section{Exploratory Variables and Follow-Up}

Exploratory variables were patient characteristics (age, sex, year of index hospitalization, insurance type [commercial, Medicaid, Medicare], comorbidities, and instrumentation and number of levels) at the index hospitalization. Comorbidity was measured with the Elixhauser Comorbidity Index (ECI) ${ }^{8}$ which was calculated based on modified ICD-9 and ICD-10 codes..$^{17}$

To calculate the look-back period and the follow-up time, we used the difference between the surgery dates and the dates of start and end enrollment times as follows:

$$
\begin{aligned}
& \text { Pre-index look-back time }= \\
& \quad \text { surgery admission date }- \text { start enrollment date } \\
& \text { Post-index follow-up time }= \\
& \quad \text { end enrollment date }- \text { surgery discharge date }
\end{aligned}
$$

\section{Index Hospitalization and Postdischarge Outcomes}

We looked at outcomes during the index hospitalization and discharge and within 1, 3, 6, 12, and 24 months after discharge. Index hospitalization outcomes were length of stay, discharge disposition, and complications (renal, cardiac, general neurosurgical, general neurological, deep vein thrombosis or pulmonary embolism, pulmonary, infection, wound infection, hydrocephalus, mechanical ventilation, laceration, or cerebrospinal fluid rhinorrhea). Postdischarge outcomes were healthcare resource use (hospital readmission rates and counts, outpatient services count, and medication refills) and their associated healthcare resource payments. For 1 month, we also evaluated emergency department (ED) visits and complications.

\section{Complications}

ICD-9 codes were used to search for complications. The incidence of complications was evaluated during the index hospitalization and 30 days after surgery. The presence of complication was noted as the occurrence of any of the complication types described above. Index hospitalization complications were complications that occurred during the index hospitalization; 1-month complications were evaluated looking at all hospitalizations and/or outpatient visits within 1 month after index hospitalization discharge.

\section{Healthcare Resource Use}

We looked at the index hospitalization and the postdis- 


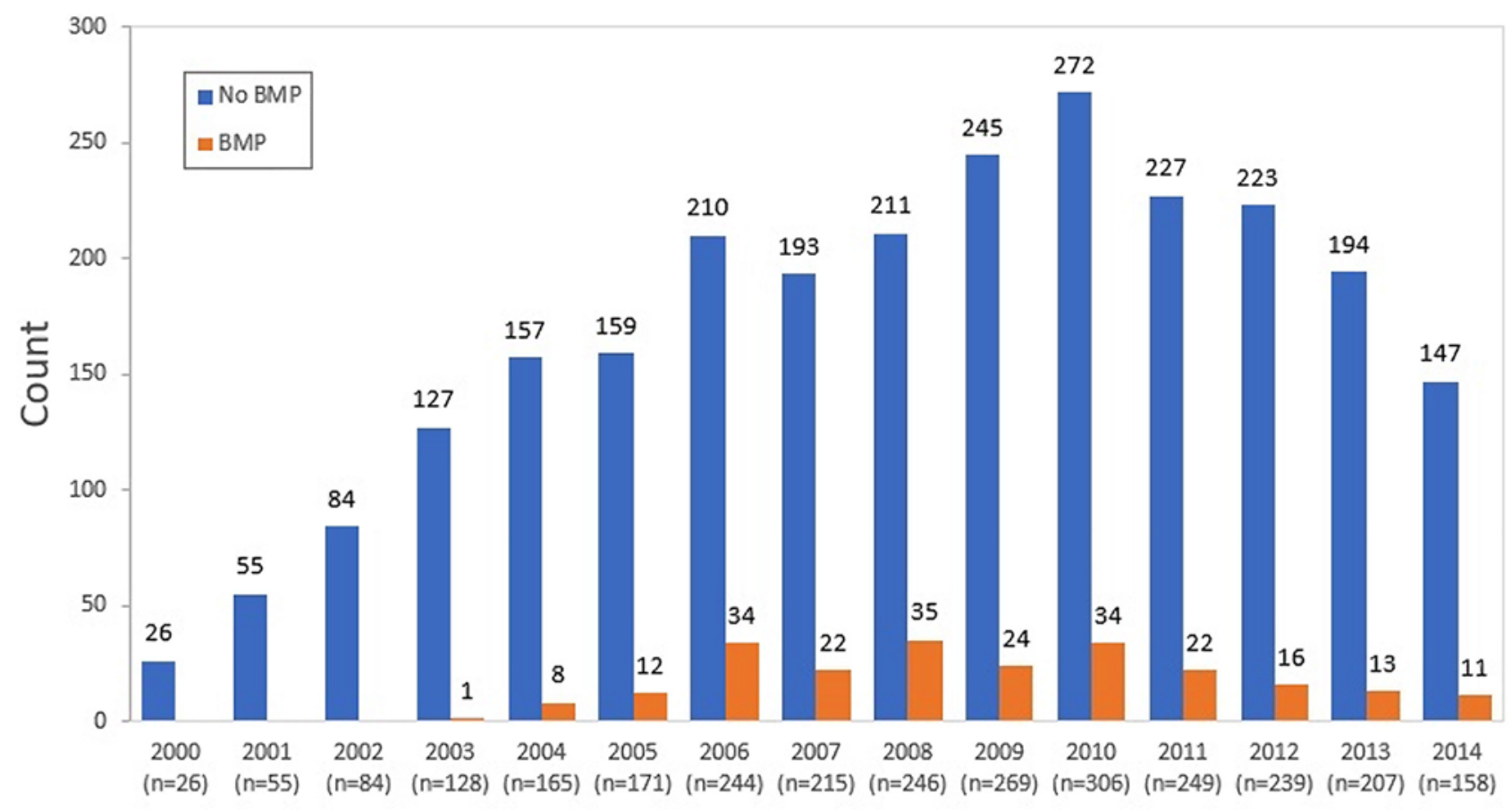

Year

FIG. 1. Bar graph showing the trends (2001-2014) in the incidence of fusion for spine infection, stratified according to the use of rhBMP-2.

charge use of healthcare resources. For the index hospitalization, we looked at the length of stay and discharge disposition. For postdischarge health resource use outcomes, we looked at 1-month ED use; hospital readmission; 1-, 3-, 6-, 12-, and 24-month hospital admissions; outpatient services; and medication refills.

\section{Healthcare Resource Payments}

We evaluated all the payments associated with the use described in the paragraph above: index hospitalization payment and postdischarge inpatient, outpatient, and medication payments. Payments were cumulated over all hospitalizations (inpatient payments), all outpatient services (outpatient payments), all prescription medication refills (medication payments), and a combination of all three. All payments were inflated to 2016 US dollars using the medical component of the Consumer Price Index, which can be accessed through the United States Bureau of Labor Statistics website. ${ }^{16}$

\section{Statistical Analysis}

Data were summarized using means and standard deviations, median and interquartile range (IQR), and the full range (minimum to maximum) for continuous variables. Categorical variables were summarized using counts and percentages. To compare the patient characteristics and outcomes between the two BMP groups, we used the Kruskal-Wallis test for continuous variables and chi-square tests for categorical variables. ${ }^{10}$ We also performed a multivariable analysis for each outcome using the linear regression on log-transformed values for continuous outcomes and the logistic regression for categorical outcomes. These models included the covariates sex, age, ECI, and insurance type, which were added in addition to the BMP use. Adjusted comparisons were presented in terms of relative risk (RR) for continuous variables and odds ratio (OR) for categorical variables. We used the software SAS 9.4 (SAS Institute Inc.) for data pre-processing and data analysis. ${ }^{19}$ All tests were 2 -sided with a 0.05 significance level.

\section{Results}

\section{Patient Demographics and rhBMP-2 Use}

Of 5274 patients who underwent fusion for spine infection identified from the database, there were $2762 \mathrm{pa}-$ tients with $>24$ months' follow-up and 3785 patients with $>12$ months' follow-up. Data from these patients are reported in this study. Based on this dataset, the incidence of rhBMP-2 use in patients undergoing fusion for spine infection has gradually increased from 2003 to 2011 and been decreasing since then (Fig. 1).

Overall, patient median age was 53 years (IQR 46-60 years). More than half $(52.43 \%, \mathrm{n}=1448)$ were female. Only $15.11 \%(\mathrm{n}=417)$ had an ECI of 3 or greater, and rhBMP-2 was used in $8.4 \%(\mathrm{n}=232)$. Patients in the rhBMP-2 group were slightly younger compared to those in the non-rhBMP-2 group (median 52 vs 53 years, $p=$ 0.015). Patients in the rhBMP-2 group had higher comorbidity indices compared to those in the non-rhBMP-2 group (ECI 2: $23.71 \%$ vs $16.5 \%$; ECI $3+: 21.55 \%$ vs 
TABLE 1. Patient demographics and characteristics stratified by rhBMP-2 use

\begin{tabular}{|c|c|c|c|c|}
\hline \multirow[b]{2}{*}{ Variables } & \multirow{2}{*}{$\begin{array}{c}\text { Combined Cohort, } \\
n=2762\end{array}$} & \multicolumn{2}{|c|}{ Fusion for Spine Infection (>24 mos follow-up) } & \multirow{2}{*}{$\begin{array}{c}\mathrm{p} \\
\text { Value }\end{array}$} \\
\hline & & Without rhBMP-2, n = 2530 (91.6\%) & With rhBMP-2, n = 232 (8.41\%) & \\
\hline Age & & & & 0.015 \\
\hline Mean (SD) & $53.2(11.7)$ & $53.4(11.6)$ & $51.5(12.4)$ & \\
\hline Median (IQR) & $53(46-60)$ & $53(46-60)$ & $52(43-59)$ & \\
\hline Range, min-max & $18-88$ & $18-88$ & $22-86$ & \\
\hline Sex: female, n (\%) & $1448(52.43 \%)$ & $1314(51.94 \%)$ & $134(57.76 \%)$ & 0.089 \\
\hline Insurance & & & & 0.012 \\
\hline Commercial, n (\%) & $2031(73.53 \%)$ & $1867(73.79 \%)$ & $164(70.69 \%)$ & \\
\hline Medicaid, $\mathrm{n}(\%)$ & $339(12.27 \%)$ & $297(11.74 \%)$ & $42(18.1 \%)$ & \\
\hline Medicare, n (\%) & $392(14.19 \%)$ & $366(14.47 \%)$ & $26(11.21 \%)$ & \\
\hline $\mathrm{ECl}$ & & & & 0.0001 \\
\hline $0, n(\%)$ & $1076(38.99 \%)$ & $1009(39.91 \%)$ & $67(28.88 \%)$ & \\
\hline $1, \mathrm{n}(\%)$ & $795(28.8 \%)$ & $735(29.07 \%)$ & $60(25.86 \%)$ & \\
\hline $2, n(\%)$ & $472(17.1 \%)$ & $417(16.5 \%)$ & $55(23.71 \%)$ & \\
\hline $3+, \mathrm{n}(\%)$ & $417(15.11 \%)$ & $367(14.52 \%)$ & $50(21.55 \%)$ & \\
\hline Region & & & & 0.0002 \\
\hline Northeast, n (\%) & $248(8.98 \%)$ & $237(9.37 \%)$ & $11(4.74 \%)$ & \\
\hline North Central, n (\%) & $691(25.02 \%)$ & $624(24.66 \%)$ & $67(28.88 \%)$ & \\
\hline South, $n(\%)$ & $962(34.83 \%)$ & $900(35.57 \%)$ & $62(26.72 \%)$ & \\
\hline West, n (\%) & $489(17.7 \%)$ & $446(17.63 \%)$ & $43(18.53 \%)$ & \\
\hline Unknown, n (\%) & $372(13.47 \%)$ & $323(12.77 \%)$ & $49(21.12 \%)$ & \\
\hline Fusion levels & & & & 0.0029 \\
\hline Cervical, n (\%) & $453(16.4 \%)$ & $431(17.04 \%)$ & $22(9.48 \%)$ & \\
\hline Thoracic/lumbar, n (\%) & $2309(83.6 \%)$ & $2099(82.96 \%)$ & $210(90.52 \%)$ & \\
\hline Corpectomy & & & & 0.021 \\
\hline Yes, n (\%) & $445(16.11 \%)$ & $420(16.6 \%)$ & $25(10.78 \%)$ & \\
\hline
\end{tabular}

Boldface type indicates statistical significance.

$14.52 \%)$. Also, commercial insurance was primarily used for both rhBMP-2 and no-rhBMP-2 cohorts. In the cohort with $>24$ months of follow-up $(n=2762), 83.6 \%$ of the patients had fusion procedures involving thoracolumbar levels, compared to only $16.4 \%$ with cervical fusion procedures (0.0029); this distribution was similar to that in the whole cohort $(\mathrm{n}=5274 ; 19.06 \%$ cervical vs $80.94 \%$ thoracic or lumbar). Also, rhBMP-2 was used in only $4.85 \%(n=22 / 453)$ of cervical fusions compared to $9.09 \%$ (210/2309) of thoracic/lumbar fusions for spine infections. This difference may be attributed to the high incidence of adverse effects related to rhBMP-2 use in cervical fusions $^{6,18}$ and the effect on clinical practice. Corpectomy was performed only in $16.11 \%$ of patients $(n=445)$ along with fusion, and rhBMP-2 was used only in a minority of these patients $(25 / 445[5.61 \%])(p=0.021$ for comparison of rhBMP-2 use between patients who had or did not have corpectomy). The Southern region (34.83\%) had the largest number of patients who underwent fusion for spinal infections (34.83\% of the overall group), whereas the Northeast had the lowest number of patients $(8.98 \%)$. Interestingly, the North Central $(28.88 \%)$ and Southern regions $(26.72 \%)$ had similar usage of rhBMP-2 during the fusion (Table 1).

\section{Outcomes at Index Hospitalization and 30 Days and 3 Months Postdischarge}

Patients in the rhBMP-2 group incurred higher costs at index hospitalization ( $\$ 60,951$ vs $\$ 47,251)$, had a higher rate of discharge to home ( $85.34 \%$ vs $77.31 \%$ ), and had lower complication rates ( $14.66 \%$ vs $18.38 \%$ ) compared to the non-rhBMP-2 group ( $\mathrm{p}<0.05)$. However, there was no significant between-groups difference in complication rates at 1 month following discharge. Also, there was no significant between-groups difference in reoperation rates at 1 month $(0.86 \%$ in the rhBMP-2 group vs $1.66 \%$ in the no-rhBMP-2 group) and 3 months (2.59\% vs $2.92 \%$, respectively). Patients in the non-rhBMP-2 group incurred higher utilization of outpatient services and medication refill costs at 1 month ( $\mathrm{p}<0.0001$ for each variable) and, in addition, also incurred higher overall payments at 3 months $(\mathrm{p}=0.0212)$ following surgery (Tables 2 and 3 , Fig. 2).

Outcomes for all patients with $>12$ months' follow-up $(n=3785)$ were also compared based on whether or not the rhBMP-2 had been used. As can be seen in Table 3, the findings were similar to those in patients with $>24$ months' follow-up. Patients who had corpectomy with decompression without rhBMP-2 incurred higher medication costs 
TABLE 2. Outcome comparison among BMP groups with > 24 months' follow-up

\begin{tabular}{|c|c|c|c|c|}
\hline \multirow[b]{2}{*}{ Variable } & \multicolumn{4}{|c|}{ Fusion for Spine Infection } \\
\hline & Without rhBMP-2, $n=2530(91.59 \%)$ & With rhBMP-2, $n=232$ (8.41\%) & p Value & Cohort, $n=2762(100 \%)$ \\
\hline \multicolumn{5}{|l|}{ Index hospitalization outcomes } \\
\hline Length of stay, median (IQR) & $3(1-7)$ & $3(2-6)$ & 0.5989 & $3(1-7)$ \\
\hline Payment, median (IQR) & $47,251(27,449-89,023)$ & $60,951(41,323-104,370)$ & $<0.0001$ & $48,981(28,118-90,086)$ \\
\hline Discharge home, $\mathrm{n}(\%)$ & $1956(77.31 \%)$ & $198(85.34 \%)$ & 0.0047 & $2152(77.99 \%)$ \\
\hline Complications, n (\%) & $465(18.38 \%)$ & $34(14.66 \%)$ & 0.1582 & $499(18.07 \%)$ \\
\hline \multicolumn{5}{|l|}{30 days postdischarge outcomes } \\
\hline Complications, n (\%) & $276(10.91 \%)$ & $28(12.07 \%)$ & 0.589 & $304(11.01 \%)$ \\
\hline ED admission, $\mathrm{n}(\%)$ & $271(10.71 \%)$ & $22(9.48 \%)$ & 0.5608 & $290(10.61 \%)$ \\
\hline \multicolumn{5}{|l|}{ Reoperation } \\
\hline New fusion, $\mathrm{n}(\%)$ & $35(1.38 \%)$ & $2(0.86 \%)$ & 0.5086 & $37(1.34 \%)$ \\
\hline Re-fusion, $\mathrm{n}(\%)$ & $12(0.47 \%)$ & $0(0 \%)$ & 0.2931 & $12(0.43 \%)$ \\
\hline At least 1 of above, $n(\%)$ & $42(1.66 \%)$ & $2(0.86 \%)$ & 0.3528 & $44(1.59 \%)$ \\
\hline \multicolumn{5}{|l|}{ Hospital readmissions } \\
\hline Readmitted, n (\%) & $196(7.75 \%)$ & $19(8.19 \%)$ & 0.8097 & $215(7.78 \%)$ \\
\hline No. of readmissions, median (IQR) & $0(0-0)$ & $0(0-0)$ & 0.8131 & $0(0-0)$ \\
\hline Payments, median (IQR) & $0(0-0)$ & $0(0-0)$ & 0.7082 & $0(0-0)$ \\
\hline \multicolumn{5}{|l|}{ Outpatient services } \\
\hline No. of services, median (IQR) & $6(2-20)$ & $5(2-20)$ & 0.5898 & $6(2-20)$ \\
\hline Payments, median (IQR) & $602(88-4110)$ & $555(89-3498)$ & 0.5981 & $600(89-4065)$ \\
\hline \multicolumn{5}{|l|}{ Medication refills } \\
\hline No. of refills, median (IQR) & $9(0-19)$ & $6(0-18)$ & 0.006 & $9(0-18)$ \\
\hline Payments, median (IQR) & $266(0-1257)$ & $78(0-845)$ & 0.0018 & $244(0-1230)$ \\
\hline Overall payments, median (IQR) & 1981 (394-6687) & $1482(348-6181)$ & 0.2836 & 1947 (390-6675) \\
\hline \multicolumn{5}{|l|}{3 mos postdischarge outcomes } \\
\hline \multicolumn{5}{|l|}{ Reoperation } \\
\hline New fusion, $\mathrm{n}(\%)$ & $61(2.41 \%)$ & $5(2.16 \%)$ & 0.807 & $66(2.39 \%)$ \\
\hline Re-fusion, $\mathrm{n}(\%)$ & $27(1.07 \%)$ & $1(0.43 \%)$ & 0.3546 & $28(1.01 \%)$ \\
\hline At least 1 of above, $n(\%)$ & $74(2.92 \%)$ & $6(2.59 \%)$ & 0.7684 & $80(2.9 \%)$ \\
\hline \multicolumn{5}{|l|}{ Hospital readmissions } \\
\hline Readmitted, n (\%) & $339(13.4 \%)$ & $29(12.5 \%)$ & 0.6997 & $368(13.32 \%)$ \\
\hline No. of readmissions, median (IQR) & $0(0-0)$ & $0(0-0)$ & 0.7106 & $0(0-0)$ \\
\hline Payments, median (IQR) & $0(0-0)$ & $0(0-0)$ & 0.881 & $0(0-0)$ \\
\hline \multicolumn{5}{|l|}{ Outpatient services } \\
\hline No. of services, median (IQR) & $23(8-55)$ & $22(8-49)$ & 0.7701 & $23(8-55)$ \\
\hline Payments, median (IQR) & $2662(648-7590)$ & $2409(607-6834)$ & 0.3397 & $2621(648-7507)$ \\
\hline \multicolumn{5}{|l|}{ Medication refills } \\
\hline No. of refills, median (IQR) & $24(6-51)$ & $20(0-44)$ & 0.0007 & $24(6-49)$ \\
\hline Payments, median (IQR) & $1364(63-4030)$ & $504(0-3171)$ & $<0.0001$ & $1279(31-3963)$ \\
\hline Overall payments, median (IQR) & $6179(2185-15,758)$ & $5080(1758-14,040)$ & 0.1828 & $6096(2152-15,518)$ \\
\hline \multicolumn{5}{|l|}{6 mos postdischarge outcomes } \\
\hline \multicolumn{5}{|l|}{ Reoperation } \\
\hline New fusion, $\mathrm{n}(\%)$ & $80(3.16 \%)$ & $6(2.59 \%)$ & 0.6289 & $86(3.11 \%)$ \\
\hline Re-fusion, $n(\%)$ & $49(1.94 \%)$ & $1(0.43 \%)$ & 0.0997 & $50(1.81 \%)$ \\
\hline At least 1 of above, $n(\%)$ & $107(4.23 \%)$ & $7(3.02 \%)$ & 0.3744 & $114(4.13 \%)$ \\
\hline \multicolumn{5}{|l|}{ Hospital readmissions } \\
\hline Admitted, n (\%) & $469(18.54 \%)$ & $38(16.38 \%)$ & 0.4164 & $507(18.36 \%)$ \\
\hline No. of readmissions, median (IQR) & $0(0-0)$ & $0(0-0)$ & 0.4824 & $0(0-0)$ \\
\hline Payments, median (IQR) & $0(0-0)$ & $0(0-0)$ & 0.6429 & $0(0-0)$ \\
\hline
\end{tabular}


» CONTINUED FROM PAGE 5

TABLE 2. Outcome comparison among BMP groups with > 24 months' follow-up

\begin{tabular}{|c|c|c|c|c|}
\hline \multirow[b]{2}{*}{ Variable } & \multicolumn{4}{|c|}{ Fusion for Spine Infection } \\
\hline & Without rhBMP-2, n = 2530 (91.59\%) & With rhBMP-2, $n=232(8.41 \%)$ & p Value & Cohort, $n=2762(100 \%)$ \\
\hline \multicolumn{5}{|l|}{6 mos postdischarge outcomes (continued) } \\
\hline \multicolumn{5}{|l|}{ Outpatient services } \\
\hline No. of services, median (IQR) & $44(18-89)$ & $46(22-84)$ & 0.5336 & $45(19-88)$ \\
\hline Payments, median (IQR) & $5248(1729-12,100)$ & $4783(1751-11,276)$ & 0.5707 & $5215(1729-12,054)$ \\
\hline \multicolumn{5}{|l|}{ Medication refills } \\
\hline No. of refills, median (IQR) & $47(12-93)$ & $35(0-84)$ & 0.0005 & $45(12-93)$ \\
\hline Payments, median (IQR) & $2804(211-8012)$ & $908(0-6072)$ & $<0.0001$ & $2714(157-7866)$ \\
\hline Overall payments, median (IQR) & $11,351(4809-26,052)$ & $10,164(3569-25,250)$ & 0.1856 & $11,234(4694-25,968)$ \\
\hline \multicolumn{5}{|l|}{12 mos postdischarge outcomes } \\
\hline \multicolumn{5}{|l|}{ Reoperation } \\
\hline New fusion, $\mathrm{n}(\%)$ & $118(4.66 \%)$ & $12(5.17 \%)$ & 0.7264 & $130(4.71 \%)$ \\
\hline Re-fusion, $\mathrm{n}(\%)$ & $93(3.68 \%)$ & $8(3.45 \%)$ & 0.8597 & $101(3.66 \%)$ \\
\hline At least 1 of above, $n(\%)$ & $166(6.56 \%)$ & $15(6.47 \%)$ & 0.955 & $181(6.55 \%)$ \\
\hline \multicolumn{5}{|l|}{ Hospital readmissions } \\
\hline Admitted, n (\%) & $657(25.97 \%)$ & $60(25.86 \%)$ & 0.9718 & $717(25.96 \%)$ \\
\hline No. of readmissions, median (IQR) & $0(0-1)$ & $0(0-1)$ & 0.9971 & $0(0-1)$ \\
\hline Payments, median (IQR) & $0(0-1247)$ & $0(0-1060)$ & 0.8858 & $0(0-1247)$ \\
\hline \multicolumn{5}{|l|}{ Outpatient services } \\
\hline No. of services, median (IQR) & $76(39-142)$ & $80(44-142)$ & 0.4046 & $76(39-142)$ \\
\hline Payments, median (IQR) & $9473(3945-19,776)$ & 8396 (3669-20,593) & 0.7841 & $9362(3931-19,922)$ \\
\hline \multicolumn{5}{|l|}{ Medication refills } \\
\hline No. of refills, median (IQR) & $87(24-174)$ & $69(0-154)$ & 0.0009 & $85(22-171)$ \\
\hline Payments, median (IQR) & $5723(595-16,297)$ & $2590(0-11,140)$ & $<0.0001$ & $5438(403-15,797)$ \\
\hline Overall payments, median (IQR) & 21,941 (9379-47,237) & $19,360(8168-43,825)$ & 0.2317 & $21,725(9212-47,004)$ \\
\hline \multicolumn{5}{|l|}{24 mos postdischarge outcomes } \\
\hline \multicolumn{5}{|l|}{ Reoperation } \\
\hline New fusion, $\mathrm{n}(\%)$ & $215(8.5 \%)$ & $17(7.33 \%)$ & 0.5385 & $232(8.4 \%)$ \\
\hline Re-fusion, n (\%) & $174(6.88 \%)$ & $18(7.76 \%)$ & 0.6135 & $192(6.95 \%)$ \\
\hline At least 1 of above, $n(\%)$ & $291(11.5 \%)$ & $26(11.21 \%)$ & 0.8926 & $317(11.48 \%)$ \\
\hline \multicolumn{5}{|l|}{ Hospital readmissions } \\
\hline Admitted, n (\%) & $975(38.54 \%)$ & $92(39.66 \%)$ & 0.7379 & $1067(38.63 \%)$ \\
\hline No. of readmissions, median (IQR) & $0(0-1)$ & $0(0-1)$ & 0.8511 & $0(0-1)$ \\
\hline Payments, median (IQR) & $0(0-16,444)$ & $0(0-16,208)$ & 0.6425 & $0(0-16,435)$ \\
\hline \multicolumn{5}{|l|}{ Outpatient services } \\
\hline No. of services, median (IQR) & $132(71-238)$ & $142(81-246)$ & 0.1984 & $133(72-238)$ \\
\hline Payments, median (IQR) & $16,552(7708-34,232)$ & $17,113(7355-34,669)$ & 0.8612 & $16,577(7673-34,357)$ \\
\hline \multicolumn{5}{|l|}{ Medication refills } \\
\hline No. of refills, median (IQR) & $168(50-327)$ & $126(0-302)$ & 0.0005 & $164(45-327)$ \\
\hline Payments, median (IQR) & $11,128(1358-32,479)$ & $5969(0-21,647)$ & $<0.0001$ & $10,521(1140-31,184)$ \\
\hline Overall payments, median (IQR) & $42,238(17,230-91,631)$ & $38,433(16,505-84,277)$ & 0.3965 & $42,074(17,154-90,819)$ \\
\hline
\end{tabular}

Boldface type indicates statistical significance. 
TABLE 3. Outcome-adjusted comparison between groups with $>24$ months $(n=2762)$ and $>12$ months $(n=3785)$ of follow-up

\begin{tabular}{|c|c|c|c|c|c|c|}
\hline \multirow[b]{2}{*}{ Variable } & \multicolumn{3}{|c|}{$>24$ Mos Follow-Up } & \multicolumn{3}{|c|}{$>12$ Mos Follow-Up } \\
\hline & $\begin{array}{c}\text { No rhBMP-2, } \\
n=2530(91.6 \%)\end{array}$ & $\begin{array}{c}\text { rhBMP-2, } \\
\mathrm{n}=232(8.41 \%)\end{array}$ & $\begin{array}{c}p \\
\text { Value }\end{array}$ & $\begin{array}{c}\text { No rhBMP-2, } \\
\mathrm{n}=3473(91.76 \%)\end{array}$ & $\begin{array}{c}\text { rhBMP-2, } \\
n=312(8.24 \%)\end{array}$ & $\begin{array}{c}p \\
\text { Value }\end{array}$ \\
\hline \multicolumn{7}{|l|}{ Index hospitalization outcomes } \\
\hline Length of stay, RR $(95 \% \mathrm{Cl})$ & Reference & $0.902(0.792-1.027)$ & 0.1183 & Reference & $0.873(0.78-0.978)$ & 0.019 \\
\hline Payment, RR (95\% CI) & Reference & $1.231(1.077-1.407)$ & 0.0024 & Reference & $1.213(1.079-1.364)$ & 0.0012 \\
\hline Discharge home, OR (95\% Cl) & Reference & $2.08(1.398-3.094)$ & 0.0003 & Reference & $1.82(1.314-2.522)$ & 0.0003 \\
\hline Complications, OR $(95 \% \mathrm{Cl})$ & Reference & $0.653(0.439-0.972)$ & 0.0355 & Reference & $0.678(0.496-0.927)$ & 0.0149 \\
\hline \multicolumn{7}{|l|}{ 30-day postdischarge outcomes } \\
\hline Complications, OR $(95 \% \mathrm{Cl})$ & Reference & $1.007(0.658-1.539)$ & 0.9761 & Reference & $0.836(0.592-1.181)$ & 0.3102 \\
\hline ED admission, OR $(95 \% \mathrm{Cl})$ & Reference & $0.723(0.45-1.162)$ & 0.1801 & Reference & $0.762(0.511-1.136)$ & 0.1824 \\
\hline \multicolumn{7}{|l|}{ Reoperation } \\
\hline New fusion, OR $(95 \% \mathrm{Cl})$ & Reference & $0.532(0.126-2.25)$ & 0.391 & Reference & $0.575(0.177-1.867)$ & 0.3575 \\
\hline Re-fusion, OR (95\% Cl) & Reference & & 0.9565 & Reference & $0.538(0.071-4.072)$ & 0.5486 \\
\hline At least 1 of above, OR $(95 \% \mathrm{Cl})$ & Reference & $0.463(0.11-1.944)$ & 0.293 & Reference & $0.475(0.147-1.53)$ & 0.2121 \\
\hline \multicolumn{7}{|l|}{ Hospital readmissions } \\
\hline Readmitted, OR (95\% Cl) & Reference & $0.947(0.574-1.562)$ & 0.8304 & Reference & $0.916(0.598-1.403)$ & 0.6876 \\
\hline No. of readmissions, $\mathrm{RR}(95 \% \mathrm{Cl})$ & Reference & $0.876(0.569-1.349)$ & 0.5484 & Reference & $0.853(0.59-1.234)$ & 0.3983 \\
\hline Payments, RR (95\% Cl) & Reference & $1.305(0.739-2.307)$ & 0.3573 & Reference & $1.127(0.693-1.832)$ & 0.6293 \\
\hline \multicolumn{7}{|l|}{ Outpatient services } \\
\hline No. of services, RR (95\% Cl) & Reference & $0.861(0.831-0.892)$ & $<0.0001$ & Reference & $0.787(0.763-0.812)$ & $<0.0001$ \\
\hline Payments, RR (95\% Cl) & Reference & $0.881(0.679-1.144)$ & 0.3422 & Reference & $0.777(0.621-0.973)$ & 0.0281 \\
\hline \multicolumn{7}{|l|}{ Medication refills } \\
\hline No. of refills, $\mathrm{RR}(95 \% \mathrm{Cl})$ & Reference & $0.823(0.791-0.856)$ & $<0.0001$ & Reference & $0.84(0.812-0.869)$ & $<0.0001$ \\
\hline Payments, RR (95\% Cl) & Reference & $0.766(0.586-1)$ & 0.0502 & Reference & $0.874(0.694-1.1)$ & 0.2512 \\
\hline Overall payments, RR (95\% Cl) & Reference & $0.783(0.612-1.001)$ & 0.0512 & Reference & $0.777(0.628-0.962)$ & 0.0206 \\
\hline \multicolumn{7}{|l|}{3 mos postdischarge } \\
\hline \multicolumn{7}{|l|}{ Reoperation } \\
\hline New fusion, OR $(95 \% \mathrm{Cl})$ & Reference & $0.823(0.325-2.089)$ & 0.6822 & Reference & $0.979(0.485-1.974)$ & 0.9522 \\
\hline Re-fusion, OR (95\% Cl) & Reference & $0.441(0.059-3.29)$ & 0.4244 & Reference & $0.885(0.315-2.488)$ & 0.8161 \\
\hline At least 1 of above, OR $(95 \% \mathrm{Cl})$ & Reference & $0.849(0.362-1.989)$ & 0.7063 & Reference & $0.956(0.506-1.807)$ & 0.8901 \\
\hline \multicolumn{7}{|l|}{ Hospital readmissions } \\
\hline Readmitted, OR (95\% Cl) & Reference & $0.82(0.54-1.244)$ & 0.3503 & Reference & $0.777(0.545-1.108)$ & 0.1639 \\
\hline No. of readmissions, $\mathrm{RR}(95 \% \mathrm{Cl})$ & Reference & $0.796(0.575-1.102)$ & 0.1693 & Reference & $0.703(0.53-0.933)$ & 0.0148 \\
\hline Payments, RR (95\% Cl) & Reference & $1.408(0.862-2.302)$ & 0.1715 & Reference & $1.158(0.772-1.737)$ & 0.4792 \\
\hline \multicolumn{7}{|l|}{ Outpatient services } \\
\hline No. of services, median (IQR) & Reference & $0.884(0.865-0.904)$ & $<0.0001$ & Reference & $0.862(0.845-0.878)$ & $<0.0001$ \\
\hline Payments, RR (95\% Cl) & Reference & $0.872(0.705-1.079)$ & 0.2082 & Reference & $0.813(0.676-0.977)$ & 0.0273 \\
\hline \multicolumn{7}{|l|}{ Medication refills } \\
\hline No. of refills, $\mathrm{RR}(95 \% \mathrm{Cl})$ & & & & Reference & $0.815(0.798-0.833)$ & $<0.0001$ \\
\hline Payments, RR (95\% Cl) & Reference & $0.947(0.735-1.22)$ & 0.6722 & Reference & $1(0.804-1.244)$ & 0.9989 \\
\hline Overall payments, RR (95\% Cl) & Reference & $0.797(0.649-0.979)$ & 0.0309 & Reference & $0.792(0.662-0.948)$ & 0.0111 \\
\hline \multicolumn{7}{|l|}{6 mos postdischarge } \\
\hline \multicolumn{7}{|l|}{ Reoperation } \\
\hline New fusion, OR $(95 \% \mathrm{Cl})$ & Reference & $0.75(0.321-1.753)$ & 0.5067 & Reference & $0.797(0.412-1.542)$ & 0.5006 \\
\hline Re-fusion, OR (95\% Cl) & Reference & $0.213(0.029-1.559)$ & 0.1279 & Reference & $1(0.628-0.428)$ & 0.4282 \\
\hline At least 1 of above, OR $(95 \% \mathrm{Cl})$ & Reference & $0.655(0.299-1.434)$ & 0.2899 & Reference & $0.733(0.401-1.34)$ & 0.3134 \\
\hline
\end{tabular}


» CONTINUED FROM PAGE 7

TABLE 3. Outcome-adjusted comparison between groups with $>24$ months $(n=2762)$ and $>12$ months $(n=3785)$ of follow-up

\begin{tabular}{|c|c|c|c|c|c|c|}
\hline \multirow[b]{2}{*}{ Variable } & \multicolumn{3}{|c|}{$>24$ Mos Follow-Up } & \multicolumn{3}{|c|}{$>12$ Mos Follow-Up } \\
\hline & $\begin{array}{c}\text { No rhBMP-2, } \\
n=2530(91.6 \%)\end{array}$ & $\begin{array}{c}\text { rhBMP-2, } \\
\mathrm{n}=232(8.41 \%)\end{array}$ & $\begin{array}{c}p \\
\text { Value }\end{array}$ & $\begin{array}{c}\text { No rhBMP-2, } \\
\mathrm{n}=3473(91.76 \%)\end{array}$ & $\begin{array}{c}\text { rhBMP-2, } \\
\mathrm{n}=312(8.24 \%)\end{array}$ & $\begin{array}{c}p \\
\text { Value }\end{array}$ \\
\hline \multicolumn{7}{|l|}{6 mos postdischarge (continued) } \\
\hline \multicolumn{7}{|l|}{ Hospital readmissions } \\
\hline Admitted, OR (95\% Cl) & Reference & $0.746(0.514-1.084)$ & 0.1245 & Reference & $0.724(0.528-0.992)$ & 0.0445 \\
\hline No. of readmissions, $\mathrm{RR}(95 \% \mathrm{Cl})$ & Reference & $0.797(0.611-1.04)$ & 0.0949 & Reference & $0.687(0.546-0.866)$ & 0.0015 \\
\hline Payments, RR (95\% Cl) & Reference & $1.475(0.944-2.304)$ & 0.0874 & Reference & $1.216(0.84-1.76)$ & 0.2988 \\
\hline \multicolumn{7}{|l|}{ Outpatient services } \\
\hline No. of services, RR (95\% Cl) & Reference & $0.913(0.898-0.929)$ & $<0.0001$ & Reference & $0.915(0.902-0.928)$ & $<0.0001$ \\
\hline Payments, RR (95\% Cl) & Reference & $0.905(0.751-1.09)$ & 0.2938 & Reference & $0.897(0.763-1.055)$ & 0.1886 \\
\hline \multicolumn{7}{|l|}{ Medication refills } \\
\hline No. of refills, RR $(95 \% \mathrm{Cl})$ & Reference & $0.786(0.772-0.801)$ & $<0.0001$ & Reference & $0.802(0.79-0.815)$ & $<0.0001$ \\
\hline Payments, RR (95\% Cl) & Reference & $0.932(0.723-1.2)$ & 0.5837 & Reference & $0.921(0.742-1.144)$ & 0.4587 \\
\hline Overall payments, RR (95\% Cl) & Reference & $0.823(0.683-0.991)$ & 0.0403 & Reference & $0.841(0.713-0.991)$ & 0.0389 \\
\hline \multicolumn{7}{|l|}{12 mos postdischarge } \\
\hline \multicolumn{7}{|l|}{ Reoperation } \\
\hline New fusion, OR (95\% Cl) & Reference & $1.051(0.567-1.947)$ & 0.8744 & Reference & $1(0.605-1.652)$ & 0.9986 \\
\hline Re-fusion, OR (95\% Cl) & Reference & $0.896(0.427-1.883)$ & 0.7721 & Reference & $0.963(0.536-1.728)$ & 0.8987 \\
\hline At least 1 of above, OR $(95 \% \mathrm{Cl})$ & Reference & $0.926(0.533-1.608)$ & 0.7851 & Reference & $0.929(0.594-1.454)$ & 0.7481 \\
\hline \multicolumn{7}{|l|}{ Hospital readmissions } \\
\hline Admitted, OR (95\% Cl) & Reference & $0.862(0.626-1.187)$ & 0.3634 & Reference & $0.802(0.609-1.056)$ & 0.1153 \\
\hline No. of readmissions, $\mathrm{RR}(95 \% \mathrm{Cl})$ & Reference & $0.889(0.729-1.085)$ & 0.2466 & Reference & $0.733(0.615-0.873)$ & 0.0005 \\
\hline Payments, RR (95\% Cl) & Reference & $1.224(0.847-1.769)$ & 0.2825 & Reference & $1.019(0.742-1.399)$ & 0.9087 \\
\hline \multicolumn{7}{|l|}{ Outpatient services } \\
\hline No. of services, RR (95\% Cl) & Reference & $0.921(0.909-0.933)$ & $<0.0001$ & Reference & $0.914(0.904-0.924)$ & $<0.0001$ \\
\hline Payments, RR (95\% Cl) & Reference & $0.95(0.8-1.128)$ & 0.5577 & Reference & $0.954(0.821-1.108)$ & 0.5341 \\
\hline \multicolumn{7}{|l|}{ Medication refills } \\
\hline No. of refills, RR $(95 \% \mathrm{Cl})$ & Reference & $0.787(0.777-0.798)$ & $<0.0001$ & Reference & $0.803(0.793-0.812)$ & $<0.0001$ \\
\hline Payments, RR (95\% Cl) & Reference & $0.871(0.673-1.127)$ & 0.2927 & Reference & $0.885(0.708-1.108)$ & 0.2868 \\
\hline Overall payments, RR (95\% Cl) & Reference & $0.849(0.711-1.014)$ & 0.0712 & Reference & $0.864(0.737-1.012)$ & 0.0693 \\
\hline \multicolumn{7}{|l|}{24 mos postdischarge } \\
\hline \multicolumn{7}{|l|}{ Reoperation } \\
\hline New fusion, OR (95\% Cl) & Reference & $0.77(0.458-1.294)$ & 0.3236 & NA & NA & NA \\
\hline Re-fusion, OR (95\% Cl) & Reference & $1.063(0.637-1.775)$ & 0.8145 & NA & NA & NA \\
\hline At least 1 of above, OR $(95 \% \mathrm{Cl})$ & Reference & $0.887(0.576-1.364)$ & 0.5839 & NA & NA & NA \\
\hline \multicolumn{7}{|l|}{ Hospital readmissions } \\
\hline Admitted, OR (95\% Cl) & Reference & $0.928(0.697-1.236)$ & 0.6088 & NA & NA & NA \\
\hline No. of readmissions, $\mathrm{RR}(95 \% \mathrm{Cl})$ & Reference & $0.934(0.808-1.079)$ & 0.3511 & NA & NA & NA \\
\hline Payments, RR (95\% Cl) & Reference & $1.014(0.755-1.363)$ & 0.9265 & NA & NA & NA \\
\hline \multicolumn{7}{|l|}{ Outpatient services } \\
\hline No. of services, RR (95\% Cl) & Reference & $0.98(0.971-0.99)$ & $<0.0001$ & NA & NA & NA \\
\hline Payments, RR (95\% Cl) & Reference & $0.992(0.842-1.169)$ & 0.9211 & NA & NA & NA \\
\hline \multicolumn{7}{|l|}{ Medication refills } \\
\hline No. of refills, RR (95\% Cl) & Reference & $0.776(0.769-0.784)$ & $<0.0001$ & NA & NA & NA \\
\hline Payments, RR (95\% Cl) & Reference & $0.813(0.624-1.058)$ & 0.1231 & NA & NA & NA \\
\hline Overall payments, RR (95\% Cl) & Reference & $0.908(0.762-1.082)$ & 0.2819 & NA & NA & NA \\
\hline
\end{tabular}

$\mathrm{NA}=$ not applicable.

Adjusted comparisons are obtained from linear contrasts obtained from multivariable regression models which include the covariates age, sex, insurance, ECl, fusion level, and vertebrectomy, in addition to BMP group. Boldface type indicates statistical significance. 


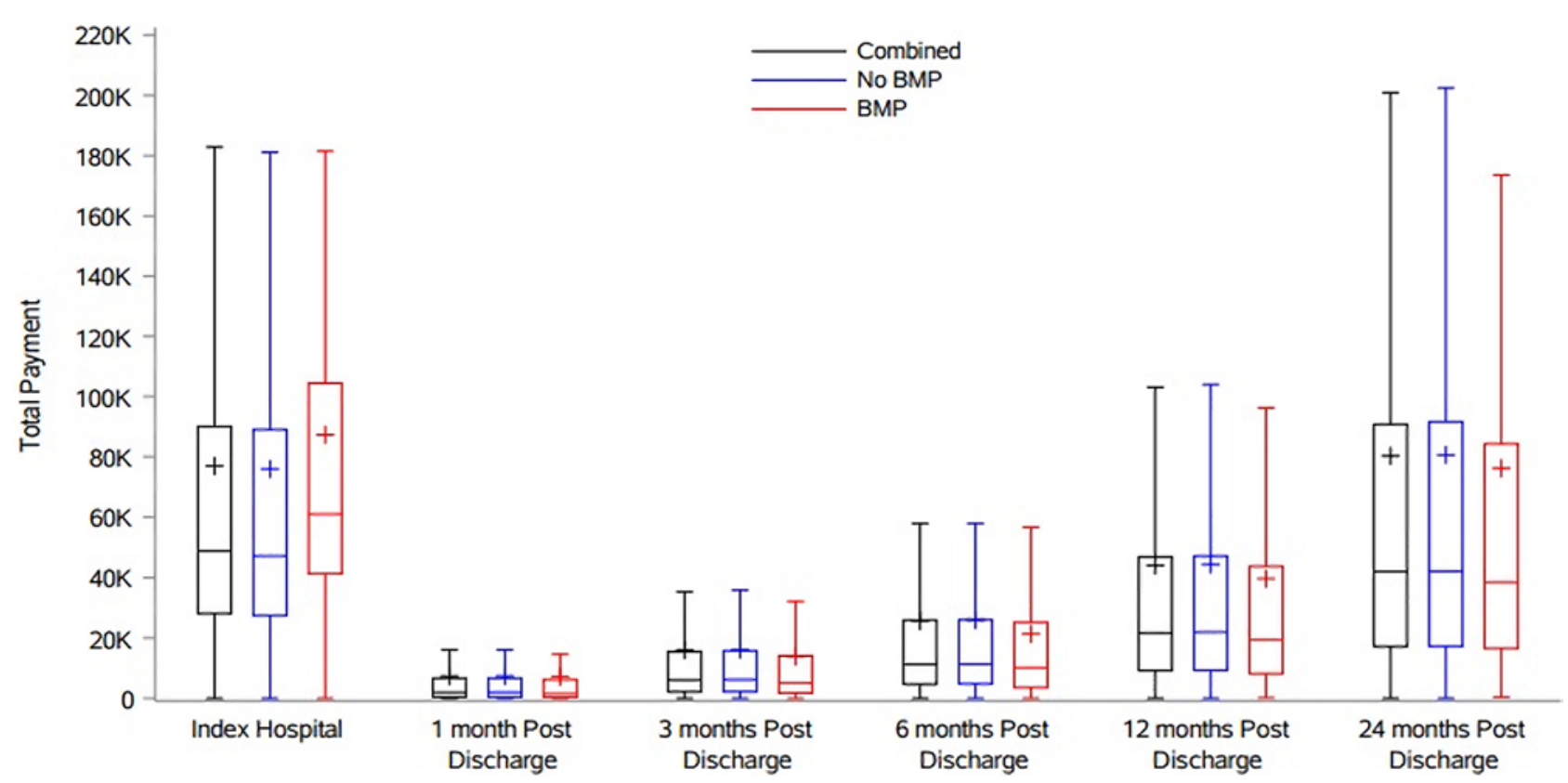

FIG. 2. Bar graph showing the total payments among patients with and without rhBMP-2 use during fusion for spine infection at index hospitalization and 1, 3,6, 12, and 24 months following the procedure.

at 1 and 3 months compared to those in whom rhBMP-2 was used ( $\mathrm{p}=0.04$ and 0.01 , respectively). No significant differences in complication and reoperation rates with use of rhBMP-2 compared to those without rhBMP-2 use in patients who underwent corpectomy with decompression and fusion at index hospitalization, 30 days, and 3 months were noted in our study $(p>0.05)$. Hospital regions had an impact on index hospitalization payments in all patients and those with > 12 and > 24 months' follow-up (Table 4).

\section{Outcomes at 6, 12, and 24 Months Postdischarge}

There was no significant difference in reoperation and hospital readmission rates at 6,12 , and 24 months between patients treated with rhBMP-2 and those who did not receive rhBMP-2. However, patients in the no-rhBMP-2 group consistently incurred higher outpatient services utilization and medication refills at 6,12 , and 24 months, compared to the rhBMP-2 group. Similarly, patients in the no-rhBMP-2 group incurred higher overall payments at 6 months only, compared to the rhBMP-2 group $(\$ 11,351$ vs $\$ 10,164 ; p=0.043$ on adjusted comparison). However, this difference in overall payments was not maintained at 12 and 24 months postdischarge $(\mathrm{p}>0.05)$ (Tables 2 and 3, Fig. 2). Also, patients who underwent corpectomy with decompression without rhBMP-2 incurred higher medication costs at 6,12 , and 24 months compared to those in whom rhBMP-2 was used $(\mathrm{p}=0.03,0.03$, and 0.01 , respectively). No other differences in outcomes were noted among the groups at these time points.

\section{Comparison Across rhBMP-2 Groups}

Adjusted comparison among rhBMP-2 groups using the whole cohort $(n=5274)$ showed that for patients who underwent fusion without rhBMP-2, length of hospital stay was longer, payments were less, complication rates were higher, and patients were discharged home (Table 5). Also, no differences were noted in terms of mortality across the groups.

\section{Discussion}

In our study, we found that thoracolumbar levels were the most common levels which underwent fusion for spine infection, corpectomy was used only in a small percentage of patients (16.11\%), and most of these patients had fusion without rhBMP-2. Overall, rhBMP-2 use was not associated with an increase in reoperation rates, and the no-rhBMP-2 group incurred higher outpatient services utilization and medication refills at 6, 12, and 24 months, compared to the rhBMP-2 group.

\section{Clinical Outcome and Complications}

O'Shaughnessy et al. ${ }^{15}$ reported on the use of rhBMP-2 in 20 patients with vertebral osteomyelitis (VO) with a mean follow-up of 20 months (range 24-53 months). In their study, lumbar spine was the most common site of $\mathrm{VO}(\mathrm{n}=11,55 \%)$ followed by thoracolumbar $(\mathrm{n}=5,25 \%)$ and lumbosacral $(\mathrm{n}=3,15 \%)$, while thoracic spine was the least common $(\mathrm{n}=1,5 \%)$. Circumferential fusion was used in $80 \%$ of patients $(\mathrm{n}=16)$ and only anterior fusion was used in the rest $(n=4,20 \%)$. Multilevel corpectomies with fusion were performed in $45 \%$ of cases, and the mean number of posterior levels fused was 6.5 (range 2-16). All patients demonstrated clinical and radiographic fusion, with no evidence of persistent/recurrent infection requiring revision surgery as of the most recent follow-up visit. 
TABLE 4. Multivariable analysis for payments during index hospitalization

\begin{tabular}{|c|c|c|c|c|c|c|}
\hline \multirow[b]{2}{*}{ Variable } & \multicolumn{2}{|c|}{ All Patients } & \multicolumn{2}{|c|}{$>12$ Mos Follow-Up } & \multicolumn{2}{|c|}{$>24$ Mos Follow-Up } \\
\hline & OR (95\% Cl) & p Value & OR (95\% Cl) & $p$ Value & OR (95\% Cl) & $p$ Value \\
\hline Age, 10-yr increase & $0.993(0.965-1.022)$ & 0.6238 & $0.995(0.962-1.03)$ & 0.782 & $1.006(0.966-1.047)$ & 0.7817 \\
\hline Sex, female vs male & $0.857(0.814-0.903)$ & $<0.0001$ & $0.848(0.797-0.902)$ & $<0.0001$ & $0.852(0.794-0.914)$ & $<0.0001$ \\
\hline \multicolumn{7}{|l|}{ Insurance } \\
\hline Medicaid vs commercial & $0.613(0.489-0.769)$ & $<0.0001$ & $0.624(0.468-0.834)$ & 0.0014 & $0.696(0.497-0.976)$ & 0.0354 \\
\hline Medicare vs commercial & $0.658(0.597-0.725)$ & $<0.0001$ & $0.653(0.583-0.731)$ & $<0.0001$ & $0.648(0.568-0.738)$ & $<0.0001$ \\
\hline \multicolumn{7}{|l|}{$\mathrm{ECl}$} \\
\hline 1 vs 0 & $1.174(1.097-1.256)$ & $<0.0001$ & $1.134(1.049-1.226)$ & 0.0015 & $1.118(1.024-1.219)$ & 0.0124 \\
\hline 2 vs 0 & $1.236(1.143-1.337)$ & $<0.0001$ & $1.25(1.14-1.371)$ & $<0.0001$ & $1.239(1.114-1.378)$ & $<0.0001$ \\
\hline $3+$ vs 0 & $1.585(1.462-1.718)$ & $<0.0001$ & $1.609(1.458-1.775)$ & $<0.0001$ & $1.681(1.496-1.889)$ & $<0.0001$ \\
\hline \multicolumn{7}{|l|}{ Regions } \\
\hline North Central vs Northeast & $0.896(0.811-0.99)$ & 0.0313 & $0.835(0.741-0.941)$ & 0.0032 & $0.852(0.743-0.976)$ & 0.0206 \\
\hline South vs Northeast & $0.834(0.759-0.917)$ & 0.0002 & $0.824(0.734-0.924)$ & $<0.0001$ & $0.883(0.774-1.006)$ & 0.061 \\
\hline West vs Northeast & $0.872(0.786-0.967)$ & 0.0096 & $0.89(0.785-1.009)$ & 0.0685 & $0.909(0.787-1.049)$ & 0.1894 \\
\hline Unknown vs Northeast & $0.778(0.62-0.977)$ & 0.0304 & $0.755(0.564-1.011)$ & 0.0589 & $0.713(0.507-1.002)$ & 0.0513 \\
\hline \multicolumn{7}{|l|}{ Fusion level } \\
\hline Thoracic vs cervical & $1.088(1.006-1.177)$ & 0.036 & $1.062(0.968-1.165)$ & 0.2054 & $0.99(0.888-1.103)$ & 0.8498 \\
\hline Lumbar vs cervical & $1.434(1.334-1.542)$ & $<0.0001$ & $1.415(1.299-1.542)$ & $<0.0001$ & $1.35(1.22-1.493)$ & $<0.0001$ \\
\hline Vertebrectomy, yes vs no & $1.312(1.221-1.409)$ & $<0.0001$ & $1.379(1.266-1.502)$ & $<0.0001$ & $1.397(1.267-1.541)$ & $<0.0001$ \\
\hline Complications, yes vs no & $2.003(1.877-2.138)$ & $<0.0001$ & $1.97(1.822-2.129)$ & $<0.0001$ & $2.143(1.947-2.358)$ & $<0.0001$ \\
\hline BMP, yes vs no & $1.218(1.106-1.342)$ & $<0.0001$ & $1.199(1.071-1.342)$ & 0.0017 & $1.225(1.077-1.393)$ & 0.002 \\
\hline
\end{tabular}

Multivariable regression includes the covariates age, sex, insurance, ECI, region, fusion level, vertebrectomy, and complications, in addition to BMP group. Boldface type indicates statistical significance.

Similarly, Aryan et al. ${ }^{3}$ reported the use of a titanium cage with rhBMP-2 (with morcellized autograft/allograft) in 15 patients following corpectomy for VO in their institutional retrospective series. The cervical spine was the region most commonly affected $(n=6,40 \%)$ followed by the thoracic $(\mathrm{n}=5,33.3 \%)$ and lumbar spine $(\mathrm{n}=4$, 26.7\%). Two-level corpectomies were performed in 13 of the 15 cases, 1 patient had a 6 -level corpectomy, and 10 patients had additional posterolateral instrumented fusion. At a mean duration of follow-up of 20 months, all patients showed radiographic evidence of fusion with no evidence of recurrent infections or complications. Similarly, in our study, we found that there was no difference in complication, reoperation, or readmission rates at $1,3,6,12$, and 24 months following surgery. Interestingly, however, we found that the rate of complications during the index hospitalization was significantly lower in the rhBMP-2 group than in the no-rhBMP-2 group (14.66\% vs $18.38 \%$; OR $0.653,95 \%$ CI $0.439-0.972, p=0.0355)$.

In subgroup analysis, we also found that among patients who underwent corpectomy with decompression and fusion there was no significant difference in complication or reoperation rates between those who were treated with rhBMP-2 versus those who did not receive rhBMP-2 when

TABLE 5. Adjusted outcome comparison among rhBMP-2 groups

\begin{tabular}{cccc}
\hline & \multicolumn{2}{c}{ Spine Fusion } & p Value \\
\cline { 2 - 3 } Variable & Without rhBMP-2 & With rhBMP-2 & \\
\hline No. of patients (\%) & $4843(91.83)$ & $431(8.17)$ & 0.2799 \\
\hline Index hospitalization outcomes & & $0.33(0.044-2.463)$ & $\mathbf{0 . 0 2 7 5}$ \\
\hline Mortality, OR $(95 \% \mathrm{Cl})$ & Reference & $0.896(0.812-0.988)$ & $<0.0001$ \\
\hline Length of stay, RR $(95 \% \mathrm{Cl})$ & Reference & $1.226(1.109-1.355)$ & $<0.0001$ \\
\hline Payment, RR $(95 \% \mathrm{Cl})$ & Reference & $1.923(1.458-2.535)$ & $\mathbf{0 . 0 0 0 4}$ \\
\hline Discharge home, OR $(95 \% \mathrm{Cl})$ & Reference & $0.613(0.468-0.804)$ & Reference \\
\hline Complications, OR $(95 \% \mathrm{Cl})$ & R & & \\
\hline
\end{tabular}

Adjusted comparisons are obtained from linear contrasts obtained from multivariable regression models which include the covariates age, sex, insurance, $\mathrm{ECl}$, fusion level, and vertebrectomy, in addition to BMP group. Boldface type indicates statistical significance. 
data were compared for any of the following: index hospitalization, 30 days postdischarge, and 3, 6, 12, and 24 months postdischarge. However, due to the small sample size of the rhBMP-2 cohort, the generalizability may be compromised and the results should be interpreted with caution.

\section{Costs and Healthcare Utilization Related to rhBMP-2 Use}

In our study, we found that patients in the rhBMP-2 group incurred lower utilization of outpatient services, medication refill costs, and overall payments at 1 and 3 months postdischarge. Also, at long-term follow-up $(6,12$, and 24 months), patients in the rhBMP-2 group had lower outpatient services utilization and fewer medication refills than those in whom rhBMP-2 was not used. In their 2007 article, Aryan et al. ${ }^{3}$ reported the cost of a small kit of Infuse rhBMP-2 (4.2 mg) as being approximately $\$ 3500$ at their institute. Use of rhBMP-2 may obviate the need to use either allograft (which may increase the chances of future infection) or autograft (which may increase the operative time and associated morbidity), but it may increase the overall costs. ${ }^{3}$ Similarly, we found that use of rhBMP-2 was associated with increased costs only at index hospitalization (median $\$ 60,951$ vs $\$ 47,251$ for the no-rhBMP-2 group, $\mathrm{p}<0.0001)$. Interestingly, patients in the rhBMP-2 group had lower overall payments at 6 months compared to the no-rhBMP-2 group (median $\$ 10,164$ vs $\$ 11,351$ ); however, this difference was not maintained at 12 and 24 months' follow-up. Also, patients in the rhBMP-2 group had significantly lower outpatient and medication refill costs, which offset the increased initial utilization cost of rhBMP-2 during index hospitalization. This may be attributed to the fact that patients who were treated with rhBMP-2 likely had increased fusion rates (as reported in previous studies) $)^{3,15}$ likely secondary to inflammatory response to rhBMP- $2^{6}$ and, therefore, decreased pain and need for medications or outpatient follow-up.

\section{Strengths and Limitations}

One of the advantages of the MarketScan database is that it is national and includes a large sample size. This allows researchers to evaluate their question with a comprehensive overview and patient population from throughout the United States. Another appealing aspect of the MarketScan database is that it reflects the full picture of healthcare utilization and payment. The data provide longitudinal information including inpatient and outpatient services, medication refills, and their associated payments. Thus, it is representative of a real-world clinical practice.

Limitations include the lack of some important clinical information considered by the physician in the decisionmaking process, such as severity and duration of the problem and imaging findings. Thus, there is possible residual bias by indication. The aggressiveness, extent of surgery, and reason for reoperation (such as hardware failure or non-union) in each individual cannot be extracted using this database; therefore, these results need to be cautiously interpreted. Also, our cohort extraction and complications definitions are based on ICD and CPT billing codes, which are prone to coding errors. Other limitations include the inability to obtain the quantity of rhBMP-2 used in each individual patient, severity of postoperative complications, complications specific to rhBMP-2 use (such as radiculitis or seroma formation), and quality of life at follow-up. Despite these limitations, our study provides insight into the US national trends of healthcare utilization and payments, comparing the use and nonuse of BMP during fusion procedures for spinal infection.

\section{Conclusions}

Following surgery for spine infection, the use of rhBMP-2 was associated with lower complication rates and higher median payments during index hospitalization compared to cases without rhBMP-2 use. There was no difference between the 2 study groups with respect to reoperation rates (index and other levels) at 3, 6, 12, or 24 months postdischarge. Patients in whom rhBMP-2 was used incurred lower utilization of outpatient services and overall payment expenditures during the same time period. We conclude that rhBMP-2 can be used safely in patients with spine infections, with cost-effective utilization of healthcare resources and without an increase in complications or reoperation rates.

\section{References}

1. Aljuboori Z, Sharma M, Altstadt T: Diffuse nocardial spinal subdural empyema: diagnostic dilemma and treatment options. Cureus 9:e1795, 2017

2. Allen RT, Lee YP, Stimson E, Garfin SR: Bone morphogenetic protein-2 (BMP-2) in the treatment of pyogenic vertebral osteomyelitis. Spine (Phila Pa 1976) 32:2996-3006, 2007

3. Aryan HE, Lu DC, Acosta FL Jr, Ames CP: Corpectomy followed by the placement of instrumentation with titanium cages and recombinant human bone morphogenetic protein-2 for vertebral osteomyelitis. J Neurosurg Spine 6:23-30, 2007

4. Brummerstedt M, Bangstrup M, Barfod TS: High mortality from pyogenic vertebral osteomyelitis: a retrospective cohort study. Spinal Cord Ser Cases 4:59, 2018

5. Cahill KS, Chi JH, Day A, Claus EB: Prevalence, complications, and hospital charges associated with use of bonemorphogenetic proteins in spinal fusion procedures. JAMA 302:58-66, 2009

6. Crawford CH III, Carreon LY, McGinnis MD, Campbell MJ, Glassman SD: Perioperative complications of recombinant human bone morphogenetic protein- 2 on an absorbable collagen sponge versus iliac crest bone graft for posterior cervical arthrodesis. Spine (Phila Pa 1976) 34:1390-1394, 2009

7. Derkinderen P, Bruneel F, Bouchaud O, Regnier B: Spondylodiscitis and epidural abscess due to Candida albicans. Eur Spine J 9:72-74, 2000

8. Elixhauser A, Steiner C, Harris DR, Coffey RM: Comorbidity measures for use with administrative data. Med Care 36:8-27, 1998

9. Kim J, Kim YS, Peck KR, Kim ES, Cho SY, Ha YE, et al: Outcome of culture-negative pyogenic vertebral osteomyelitis: comparison with microbiologically confirmed pyogenic vertebral osteomyelitis. Semin Arthritis Rheum 44:246252,2014

10. Littell RC, Stroup WW, Freund RJ: SAS for Linear Models, ed 4. Cary, NC: SAS Institute, 2002

11. Longardner K, Allen A, Ramgopal M: Spinal osteomyelitis due to Mycobacterium fortuitum in a former intravenous drug user. BMJ Case Rep 2013:bcr2013010326, 2013

12. Lora-Tamayo J, Euba G, Narváez JA, Murillo O, Verdaguer 
$\mathrm{R}$, Sobrino B, et al: Changing trends in the epidemiology of pyogenic vertebral osteomyelitis: the impact of cases with no microbiologic diagnosis. Semin Arthritis Rheum 41:247255, 2011

13. Mulconrey DS, Bridwell KH, Flynn J, Cronen GA, Rose PS: Bone morphogenetic protein (RhBMP-2) as a substitute for iliac crest bone graft in multilevel adult spinal deformity surgery: minimum two-year evaluation of fusion. Spine (Phila Pa 1976) 33:2153-2159, 2008

14. Mylona E, Samarkos M, Kakalou E, Fanourgiakis P, Skoutelis A: Pyogenic vertebral osteomyelitis: a systematic review of clinical characteristics. Semin Arthritis Rheum 39:1017,2009

15. O'Shaughnessy BA, Kuklo TR, Ondra SL: Surgical treatment of vertebral osteomyelitis with recombinant human bone morphogenetic protein-2. Spine (Phila Pa 1976) 33:E132E139, 2008

16. Parikh AA, Robinson J, Zaydfudim VM, Penson D, Whiteside MA: The effect of health insurance status on the treatment and outcomes of patients with colorectal cancer. J Surg Oncol 110:227-232, 2014

17. Quan H, Sundararajan V, Halfon P, Fong A, Burnand B, Luthi JC, et al: Coding algorithms for defining comorbidities in ICD-9-CM and ICD-10 administrative data. Med Care 43:1130-1139, 2005

18. Shields LB, Raque GH, Glassman SD, Campbell M, Vitaz $\mathrm{T}$, Harpring J, et al: Adverse effects associated with highdose recombinant human bone morphogenetic protein-2 use in anterior cervical spine fusion. Spine (Phila Pa 1976) 31:542-547, 2006

19. Stokes M, Davis C, Koch G: Categorical Data Analysis Using the SAS System, ed 2. Cary, NC: SAS Institute, 2000
20. Swiontkowski MF, Aro HT, Donell S, Esterhai JL, Goulet J, Jones A, et al: Recombinant human bone morphogenetic protein-2 in open tibial fractures. A subgroup analysis of data combined from two prospective randomized studies. J Bone Joint Surg Am 88:1258-1265, 2006

\section{Disclosures}

The authors report no conflict of interest concerning the materials or methods used in this study or the findings specified in this paper.

\section{Author Contributions}

Conception and design: Sharma. Acquisition of data: Ugiliweneza. Analysis and interpretation of data: Sharma. Drafting the article: Sharma, Alhourani. Critically revising the article: Sharma, Dietz, Alhourani, Ugiliweneza, Wang. Reviewed submitted version of manuscript: Sharma, Dietz, Ugiliweneza, Wang, Drazin. Approved the final version of the manuscript on behalf of all authors: Boakye. Statistical analysis: Ugiliweneza, Wang. Administrative/technical/material support: Boakye, Drazin. Study supervision: Boakye.

\section{Correspondence}

Maxwell Boakye: University of Louisville, School of Medicine, Louisville, KY. maxwell.boakye@ulp.org. 